\title{
Hydrophobic interactions of peptides with membrane interfaces
}

\author{
Stephen H. White ${ }^{*}$, William C. Wimley ${ }^{1}$ \\ Department of Physiology and Biophysics, University of California, Irvine, CA 92697-4560, USA
}

Received 4 December 1997; received in revised form 16 February 1998; accepted 16 February 1998

\begin{abstract}
The thermodynamic principles underlying the structural stability of membrane proteins are difficult to obtain directly from whole proteins because of intractable problems related to insolubility in the aqueous phase and extreme stability in the membrane phase. The principles must therefore be surmised from studies of the interactions of small peptides with lipid bilayers. This review is concerned with the hydrophobic interactions of such peptides with the interfacial regions of lipid bilayers. We first develop a general framework for thinking about the thermodynamics of membrane protein stability that centers on interfacial interactions and review the structural and chemical evidence that supports this interface-centered point of view. We then describe an experimentally determined whole-residue interfacial hydrophobicity scale that reveals the central role of the peptide bond in partitioning and folding. Finally, we consider the complexity and diversity of interfacial interactions revealed by differences between side-chain hydrophobicities determined using different classes of peptides. (C) 1998 Elsevier Science B.V. All rights reserved.
\end{abstract}

Keywords: Hydrophobicity scale; Lipid bilayer; Lipid bilayer structure; Peptide partitioning

\section{Contents}

1. Introduction $\ldots \ldots \ldots \ldots \ldots \ldots \ldots \ldots \ldots \ldots \ldots \ldots \ldots \ldots \ldots \ldots \ldots$

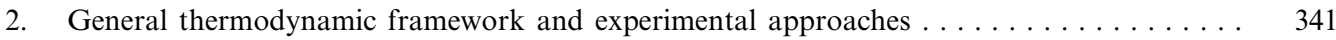

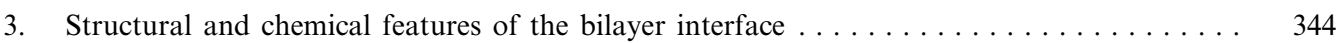

4. An experimentally determined whole-residue interfacial hydrophobicity scale . . . . . . 346

4.1. Experimental approach to the determination of the interfacial free energy scale . . . 346

4.2. General features of the interface revealed by the interfacial free energy scale . . . . . . 347

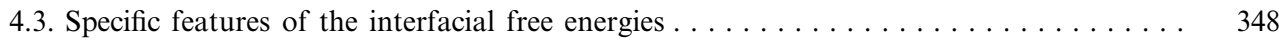

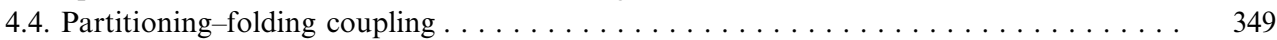

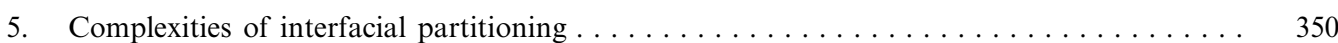

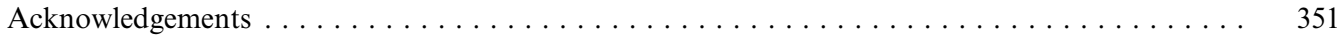

\footnotetext{
* Corresponding author. Fax: +1 (714) 824-8540; E-mail: shwhite@uci.edu

1 Present address: Department of Biochemistry, Tulane University Medical Center, New Orleans, LA 70122-2699, USA.
} 


\section{Introduction}

A great deal is now known about the general principles of the folding and structural stability of soluble proteins [1,2]. Although we are far from understanding folding to the extent that the structure of soluble proteins can be predicted from sequence, there is at least a large amount of useful and informative experimental data [3-5]. The state of affairs for membrane proteins is comparatively grim. Some general principles are beginning to emerge [6-9], but the amount of thermodynamic data is quite limited due primarily to the experimental difficulties caused by their insolubility in water in both their folded and unfolded forms and their great stability in the membrane. The general approach to the thermodynamic stability of proteins is to examine the energetics of the folding/unfolding process [10] induced by heat [11] or denaturants [12]. This approach, which requires that the folding/unfolding reaction be reversible, has not been particularly useful for membrane proteins. As discussed by Haltia and Freire [13], the main difficulty is that membrane proteins resist complete denaturation because of the great stability of the transmembrane secondary structure elements within the membrane. The denaturation that does occur is frequently irreversible and arises largely from the dissociation of multimers, subunits, and secondary structure elements and the unfolding of extramembrane domains. The stability of the individual secondary structure elements, usually $\alpha$-helices, is revealed by the large amount of secondary structure (determined by circular dichroism) that remains after denaturation. This residual structure within the membrane phase (bilayers or micelles) indicates the resistance of secondary structure elements to desorption and/or unfolding under denaturing conditions.

The observed stability of transmembrane helices is consistent with the very rough value of about -70 $\mathrm{kcal} / \mathrm{mol}$ estimated by Engelman et al. [14]. The main source of this stability arises from the high cost of breaking H-bonds in non-polar environments. The cost of partitioning a peptide bond $(\mathrm{CONH})$ into $\mathrm{CCl}_{4}$ from water has been estimated by Roseman [15] to be $\sim 6 \mathrm{kcal} / \mathrm{mol}$ when not $\mathrm{H}$-bonded, but only $0.6 \mathrm{kcal} / \mathrm{mol}$ when H-bonded. These values suggest a cost of $\sim 100 \mathrm{kcal} / \mathrm{mol}$ for unfolding a helix of 20 amino acids within a non-polar environment. More important for the present discussion, they indicate that the energetics of peptide bond partitioning and $\mathrm{H}$-bond formation play a dominant role in the structural stability of membrane proteins.

The free energy cost of 'desorbing' a transmembrane $\alpha$-helix into the aqueous phase, which has never been satisfactorily determined experimentally, will be greatly affected by the cost of partitioning $\mathrm{H}$ bonded peptide bonds from water to membrane. For the bilayer insertion of a 20 AA non-polar $\alpha$-helix, the free energy reduction available from the hydrophobic effect ranges from $\sim 15 \mathrm{kcal} / \mathrm{mol}$ for polyalanine to $\sim 45 \mathrm{kcal} / \mathrm{mol}$ for poly-leucine. These values will be countered by the cost of partitioning H-bonded peptide bonds ( $\left.\Delta G_{\text {Hbond }}\right)$. If Roseman's estimate [15] for $\Delta G_{\mathrm{Hbond}}$ of about $0.6 \mathrm{kcal} / \mathrm{mol}$ per $\mathrm{H}$-bonded $\mathrm{CONH}$ holds for membranes, the favorable hydrophobic free energies of transfer would be reduced by $\sim 12 \mathrm{kcal} / \mathrm{mol}$. The insertion of both the alanine and the leucine helices would be favored in that case. However, recent calculations by Ben-Tal et al. [16] suggest that $\Delta G_{\mathrm{Hbond}}$ may be as high as $\sim 1.5 \mathrm{kcal} / \mathrm{mol}$ per residue or $30 \mathrm{kcal} / \mathrm{mol}$ for a 20 AA helix. In that case, the poly-alanine helix would be highly disfavored while the partitioning of the poly-leucine helix would remain quite favorable. These considerations indicate, again, the importance of the energetics of CONH partitioning and $\mathrm{H}$-bond formation and emphasize the need for experimentally determined values. An accurate value for $\Delta G_{\text {Hbond }}$ is exceptionally important because it essentially determines the 'decision threshold' for transmembrane helix prediction in hydropathy plot analyses [17].

Most estimates of the energetics of helix stability in membranes rely upon measurements derived from bulk-phase partitioning measurements. But, lipid bilayer membranes are not equivalent to bulk solvents. They are more like soluble proteins in that they exist in a free energy minimum determined by small differences between very large enthalpic and entropic terms. As a result, free energies of partitioning mol- 
ecules of any sort into membranes must include inevitable changes in bilayer free energy resulting from bilayer perturbations. We have referred to these changes collectively as the 'bilayer effect' [18] to distinguish them from the hydrophobic effect which arises solely from the water due to the energetic consequences of removing non-polar solutes. Bilayer effects are revealed by the significant differences in the thermodynamics of partitioning of a non-polar solute from water into bilayers and into bulk phases [18-22]. We surmised from measurements of the partitioning of indole compounds from water to cyclohexane and bilayers that the bilayer effect can contribute significantly to the free energy of partitioning $[18,23,24]$. This indicates the unreliability of bulkphase measurements alone as a means for understanding membrane protein thermodynamics. Because there is presently no simple way for correcting bulk-phase data for the bilayer effect, the only reliable method for determining membrane protein thermodynamic parameters is by direct measurements on bilayer systems which inherently include the bilayer effect.

The major difficulty with direct thermodynamic measurements on membrane proteins in bilayers, beyond those discussed by Haltia and Freire [13], is their general intractability arising from insolubility in the aqueous phase. One way, perhaps the only way, to avoid this difficulty is to study the interactions of small hydrophobic peptides (3-30 residues) with lipid bilayers in a systematic fashion. Our general strategy, based upon the approach of Jacobs and White [25], uses a combination of structural and thermodynamic measurements of the interactions of small host-guest hydrophobic peptides with lipid bilayers. Broadly, the strategy is to answer four questions: (1) What structures do the peptides adopt; (2) what is the transbilayer location of the peptides; (3) what is the energetic cost of getting there; and (4) what changes occur in the bilayer structure as a result? We summarize in this review our progress using this strategy. The review is sharply focused on hydrophobicity-driven interactions at bilayer interfaces, but electrostatic interactions are equally important. The recent work of McLaughlin and Honig and their colleagues should be consulted for the latest information about interfacial electrostatic interactions (see [26]).

\section{General thermodynamic framework and experimental approaches}

Fig. 1 shows a thermodynamic cycle for the fold-

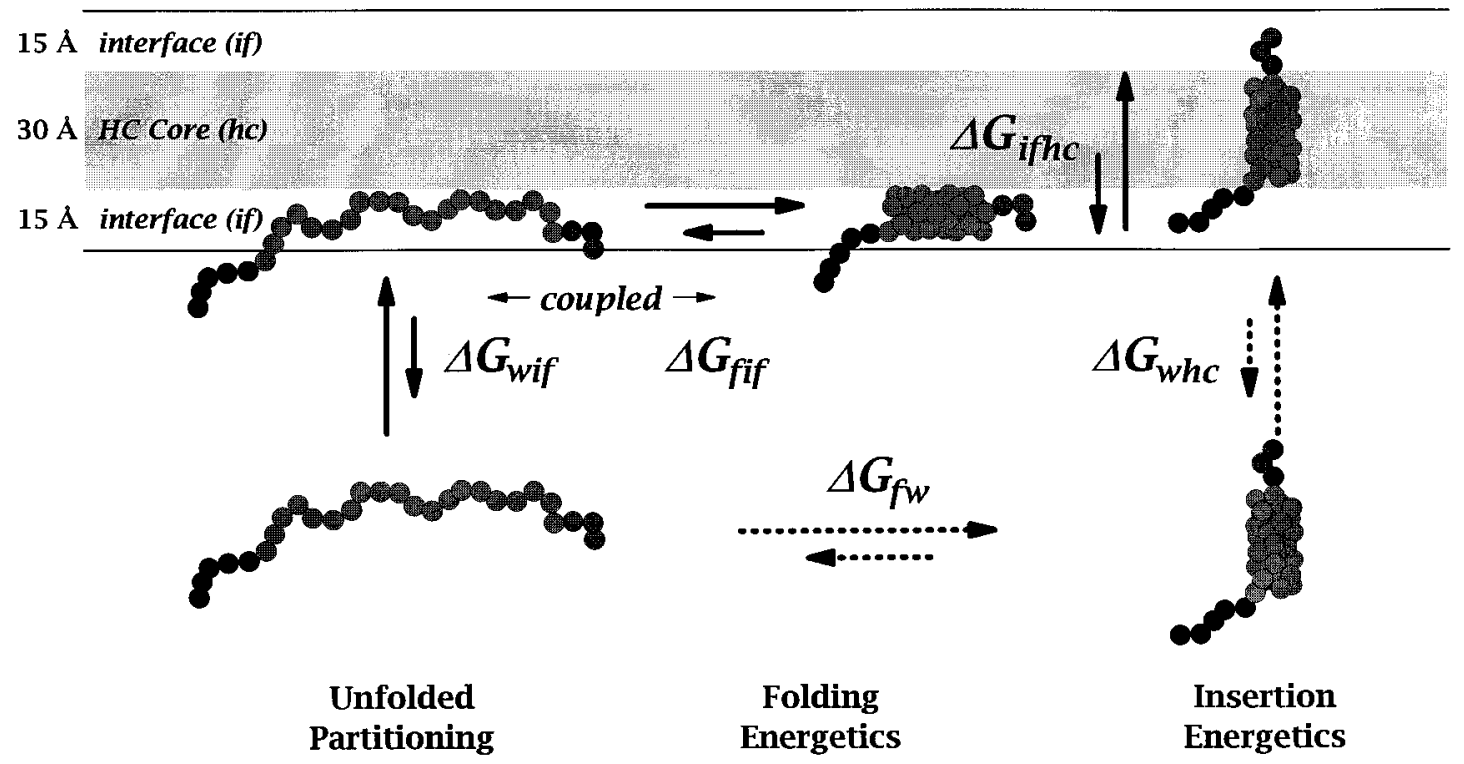

Fig. 1. A general framework for describing the thermodynamics of the interactions of peptides and proteins with lipid bilayers that recognizes the importance of the interfacial regions (Fig. 2). The scheme and its rationale are described in detail in (Section 2). 
ing and bilayer insertion of a small hydrophobic peptide that forms an $\alpha$-helix. The bilayer is subdivided into two $\sim 15 \AA$ thick interfacial (if) regions and the $\sim 30 \AA$ thick hydrocarbon core (hc) based on the membrane diffraction studies of Wiener and White [27]. The interfaces and hydrocarbon core are treated as distinct 'phases' for reasons outlined in the next section. Two pathways for insertion are shown: a direct 'water' path (lower half of the cycle, dotted arrows) and an indirect 'interfacial' path (upper half of the cycle, solid arrows). The free energy of folding and insertion along the water path is composed of the free energy change $\Delta G_{\mathrm{fw}}$ for folding (f) from random coil to $\alpha$-helix in water (w) and the change $\Delta G_{\mathrm{whc}}$ for the insertion of the into the hydrocarbon core. The free energy of folding and insertion along the interfacial path is composed of three terms: the partitioning of the unfolded chain from water to interface $\Delta G_{\text {wif }}$, the folding in the interface $\Delta G_{\text {fif }}$, and the insertion of the folded peptide from the interface to the hydrocarbon core, $\Delta G_{\text {ifhc }}$. Both bilayer-insertion paths in Fig. 1 are based upon the assumption that the inserted peptide exists in the aqueous phase as a monomer. Because there is a very strong tendency for non-polar peptides to form aggregates, this critical assumption can be a difficult one to satisfy. Before adopting it, one must verify experimentally that the peptide is in fact monomeric.

There have been three attempts to measure the free energy of insertion, $\Delta G_{\mathrm{whc}}$, but with mixed success [28-30]. Moll and Thompson [28] attempted to measure $\Delta G_{\mathrm{whc}}$ along the water path, and reported a value of about $-5 \mathrm{kcal} / \mathrm{mol}$. Their experiments were based upon measurements of the partitioning of a 20-residue alanine peptide that was covalently linked to bovine pancreatic trypsin inhibitor (BPTI). The reliability of this measurement is uncertain for three reasons. First, the conformation of the $\mathrm{Ala}^{20}$ peptide in the aqueous phase is uncertain. Although a polyalanine peptide of this length is likely to have a high helix content [3], it is not clear whether the measurement was of $\Delta G_{\mathrm{whc}}$ or $\Delta G_{\mathrm{whc}}+\Delta G_{\mathrm{fw}}$ because the helix content of the $\mathrm{Ala}^{20}$ peptide was not reported. Second, $\mathrm{Ala}^{20}$ alone is likely to be highly insoluble, at least as a monomer. This raises the possibility that the $\mathrm{Ala}^{20}$ domain may associate non-covalently in some way with the BPTI carrier, further confusing the meaning of the reported free energy. Third, no evidence was presented that the $\mathrm{Ala}^{20}$ domain actually crossed the lipid bilayer, was adsorbed only on the surface, or was distributed between surface and transmembrane locations. Despite these uncertainties and in the light of the earlier discussion (Section 1$),-5 \mathrm{kcal} / \mathrm{mol}$ is not an unreasonable value of $\Delta G_{\text {whe }}$ for $\mathrm{Ala}^{20}$.

Soekarjo et al. [29] showed that the insertion of M13 procoat protein into bilayers follows the interfacial path and cleverly determined free energies associated with the process. They found that M13 procoat, containing two putative transmembrane domains, was distributed $80 \%$ in the interface and $20 \%$ across the bilayer based upon proteinase $\mathrm{K}$ digestion experiments. They concluded that $\Delta G_{\mathrm{whc}}=-14.8 \mathrm{kcal} / \mathrm{mol}$ for the presumed two-helix hairpin and estimated that $\Delta G_{\mathrm{whc}}=-12 \mathrm{kcal} / \mathrm{mol}$ for a single helix. Although these experiments represent an excellent attempt to measure $\Delta G_{\text {whc }}$, they suffer from a failure to determine the secondary structure of procoat in either the aqueous or membrane phases or to establish with certainty that the proteinase-resistant population of procoat was truly across the membrane. Therefore, uncertainty remains about exactly what free energies terms are represented by their value for $\Delta G_{\mathrm{whc}}$.

The most recent attempt to measure $\Delta G_{\text {whc }}$ is that of Hunt et al. [30,31] who found that a 36-residue peptide containing the $\mathrm{C}$ helix of bacteriorhodopsin (bR) could be caused to insert spontaneously into lipid bilayers as a transmembrane $\alpha$-helix by lowering $\mathrm{pH}$ in order to protonate one of the two Asp residues located within the known transmembrane domain of bR $[32,33]$. The insertion followed an interfacial pathway because Hunt et al. [30] found that the C-helix peptide binds to the surface of DMPC vesicles in a largely unfolded form at $\mathrm{pH} 7.8$ (Asp deprotonated), but inserts reversibly upon lowering the $\mathrm{pH}$. From their data, Hunt et al. [30] estimated a value for the free energy of insertion of about $-6 \mathrm{cal} / \mathrm{mol}$. This value probably does not correspond to $\Delta G_{\mathrm{whc}}$ because the peptide was neither helical nor monomeric the aqueous phase. If the peptide had been unfolded, but monomeric, their measurement would have been of $\Delta G_{\mathrm{fw}}+\Delta G_{\mathrm{whc}}$, a valuable number to have. Unfortunately, the aggregation problem, as Hunt et al. recognized, makes the mean- 
ing of their value for the free energy of insertion problematic.

These three attempts to measure $\Delta G_{\mathrm{whc}}$ [28-30] show that the general thermodynamic cycle of Fig. 1 is reasonable and appropriate. But, they also remind us of how difficult it is to measure the free energy terms of the cycle. A strategic difficulty revealed by the experiments is that studies of very insoluble peptides such as $\mathrm{Ala}^{20}$ or very complex ones such as 73-residue M13 procoat, are difficult to interpret as well as perform. We have therefore adopted a 'bootstrap' strategy for the purpose of measuring the free energy of folding and insertion along the interfacial path. The first step of the strategy is to determine the interfacial partitioning of well-characterized small peptides (three to seven residues) that have no secondary structure in the aqueous or interfacial phases $[25,34,35]$. This step provides two advantages.

First, one can obtain an interfacial hydrophobicity scale that can be used for calculating $\Delta G_{\text {wif }}$ for any soluble random-coil peptide. This calculated value may or may not, however, be the same as a measured value for a truly random-coil peptide larger than five or so peptides. Nevertheless, the value is useful because it at least provides a virtual reference state for the sorting out the multitude of factors that determine partitioning. We therefore designate the waterto-interface free energy change calculated from the small-peptide interfacial hydrophobicity scale as $\Delta G_{\text {wif }}^{\mathrm{v}}$ to indicate its status as a virtual reference value. This value is exceptionally important for peptides such as melittin $[35,36]$ for which there is a strong coupling (Fig. 1) between partitioning and folding (see below). The population of unfolded interfacial peptide is so small in such cases that it cannot be detected. The ability to calculate $\Delta G_{\text {wif }}^{\mathrm{v}}$ permits $\Delta G_{\text {fif }}$ to be estimated. The second advantage of the first step of our bootstrap strategy is that one has a starting set of thermodynamic parameters that is invaluable for designing more complex (longer) peptides. For example, the limited number of hydrophobicity values determined by Jacobs and White [25] for their A-W-A-O-t-butyl peptides made it possible to arrive quickly at the design of the AcWL-X-LL peptides used for the experimental determination of a complete whole-residue hydrophobicity scale [34].
The second step of our bootstrap strategy is to design and synthesize longer peptides that can, theoretically, fold and insert spontaneously. Experiments with such peptides provide vital information about the behavior of longer hydrophobic peptides in both water and membranes. Our experience to date suggests that the biggest single problem is multimer formation in the aqueous phase. Although some large ( $\sim 30$ residues) peptides appear to be soluble because 'clear' aqueous solutions are obtained, they actually form soluble multimers (see [37] for a discussion of this issue). The working out of the details of the thermodynamic cycle of Fig. 1 demands that there be a set of conditions for which there is a measurable concentration of monomers in the aqueous phase and for which there are transmembrane helices. If those conditions can be achieved, then one must carefully establish the relative amounts of unfolded and folded peptides in interface and the fraction of folded peptide that is transbilayer. The two most critical experiments are to prove that the peptide is monomeric in the aqueous phase and that presumed transmembrane peptides are truly transmembrane.

Deber and colleagues $[38,39]$ have approached the folding of proteins in membranes with a different bootstrap approach by measuring the helicity of cationic host-guest peptides of the form SKSKAXAXAWAXAKSKSKS bound to anionic micelles or vesicles. These experiments address $\Delta G_{\text {fif }}$ directly (see Fig. 1). Interestingly, they found a wide range of helical propensities for the guest amino acids in membranes that are very different from those for peptides in aqueous solution. Membrane helical propensities in their system are roughly correlated with hydrophobicity, but were shown to be contextand position-dependent as well [39]. Blondelle et al. [40] have taken a similar approach, but with somewhat different results, using the host-guest peptide Ac-KYA $\mathrm{XA}_{5} \mathrm{~K}-\mathrm{NH}_{2}$. Through the use of these bootstrap experiments, the energetics of protein folding in membranes are beginning to be defined. But, interactions in the bilayer interface are complex. An important part of the problem of unraveling the complexities is to understand the structure of fluid bilayers, and particularly the bilayer interface. 

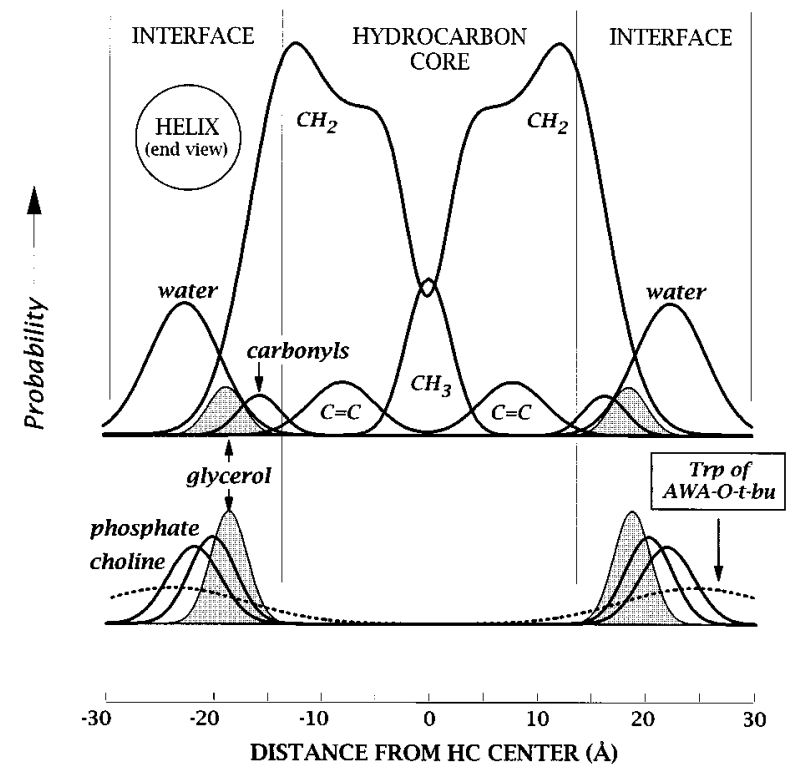

Fig. 2. The structure of a fluid dioleoylphosphocholine (DOPC) bilayer determined by the joint refinement of X-ray and neutron diffraction data [27]. The 'structure' consists of the time-averaged distributions of the principal structural groups of the lipid projected onto an axis normal to the bilayer plane. The distributions are constructed from Gaussian distributions whose areas equal the number of structural groups represented by the Gaussians; the distributions therefore represent the probability of finding a structural group at a particular location. The interfaces of the bilayer are defined as the regions occupied by headgroup water of hydration. Notice that an $\alpha$-helix that is parallel to the bilayer can be comfortably accommodated in the interfaces. Also shown is the distribution of the tryptophan (Trp) of Ala-Trp-Ala-O-tert-butyl [25] partitioned into the bilayer (see Section 3). The figure is modified from that of White [17].

\section{Structural and chemical features of the bilayer interface}

The structural evidence for the division of the bilayer into interfacial and hydrocarbon-core phases, shown in Fig. 2, is based upon the liquid-crystallographic determination of the structure of fluid $\left(\mathrm{L}_{\alpha}\right.$ phase) dioleoylphosphatidylcholine (DOPC) bilayers performed by Wiener and White [27,41-45] (reviewed in $[46,47])$. Fluid bilayers are biologically relevant because cellular membranes must be in a fluid state for cells to function. But, their high thermal disorder excludes completely the possibility of atomic-resolution three-dimensional crystallographic images that can be obtained from crystallized membrane lipids $[48,49]$. Multilamellar bilayers obtained from phos- pholipids by dispersal in water or deposition on surfaces, liquid crystals, are highly periodic in a direction normal to the bilayers, but highly thermally disordered in the bilayer plane. The resulting onedimensional crystallinity allows one to examine the distribution of matter along the bilayer normal by diffraction methods. The 'structure' of a fluid bilayer is consequently defined operationally as the timeaveraged spatial distributions of the principal structural (quasi-molecular) groups of the lipid (carbonyls, phosphates, etc.) projected onto an axis normal to the bilayer plane [41]. Complete and fully resolved bilayer structures comprised of the collection of water and structural-group distributions can be determined through the joint refinement of X-ray and neutron diffraction data obtained by using lipids that have been labeled at specific sites with heavy metals $[43,50,51]$ or deuterium $[44,52,53]$.

The time-averaged transbilayer distributions of the quasi-molecular groups shown in Fig. 2 can be thought of as probability or number densities. That is, they give the probability of finding a particular structural group at a specific location in the bilayer or the number of groups per unit volume at a given location. The areas under the peaks are equal to the number of structural groups per lipid molecule (e.g. two methyls, 28 methylenes, one phosphate). The peaks are determined in the liquid-crystallographic experiment with high precision $(0.02-0.5 \AA)$, represent fully resolved [41] images, and are accurate representations of the true thermal motion of the molecules. This thermal motion is a fundamental and important feature of fluid bilayers that plays a critical role in peptide-bilayer interactions. Although the structural image of Fig. 2 was obtained at low hydration, 5.4 waters per lipid, recent work in our laboratory demonstrates that the overall structure changes in only subtle ways as the water content is increased [54].

The complexity of bilayers as 'non-polar' phases for peptide partitioning is apparent in Fig. 2. The image shows that the fluid bilayer can be naturally divided (vertical lines) into interfacial and hydrocarbon core regions based upon the distribution of the water of hydration of the headgroup. The hydrocarbon region and the combined interfacial regions each have a width of about $30 \AA$ so that the interfaces account for $50 \%$ of the total thermal thickness of the 
bilayer. This emphasizes the fact that the bilayer should not be treated simply as a thin hydrocarbon slab separating two aqueous phases. Of particular importance for interfacial partitioning is the fact that the interfaces consist of a complex mixture of water and phosphocholine, glyceryl, carbonyl, and methylene groups. These regions are rich in possibilities for non-covalent interactions with peptides. Furthermore, as shown in Fig. 2, the $15 \AA$ thermal thickness of an interface can comfortably accommodate an $\alpha$-helix (diameter $\approx 10 \AA$ ) running parallel to the bilayer plane. Included in Fig. 2 is the transbilayer distribution of the Trp residue of the small peptide A-W-A-O-t-butyl determined by neutron diffraction [25]. Even though partitioning of this peptide is driven primarily by the hydrophobic effect [25], it is, nevertheless, located almost entirely in the interfacial region. The fact that the Trp is found largely in the same region as the phosphocholine group has important implications for the assembly and stability of membrane proteins [18] (see below). For present purposes, however, the most important conclusion is that small hydrophobic peptides which cannot form secondary structure (i.e. form intramolecular $\mathrm{H}$ bonds) must be restricted to the interface because of the energetic cost of partitioning the peptide bonds into the hydrocarbon core [25].

Besides being chemically heterogeneous, the interfaces are, not surprisingly, regions in which dramatic changes in polarity occur over small distances. To illustrate this point, we show in Fig. 3 (heavy line) the 'polarity profile' of a DOPC bilayer derived from the structural image of Fig. 2. This profile was calculated from the quasi-molecular-group volumes of Wiener and White [27] and the partial charges of the groups using the atomic partial charges of phospholipids reported by Charifson et al. [55]. It represents the average density of atomic partial charges, both + and - , calculated by weighting the absolute values of the charge densities by the number density and group volume at each position across the bilayer. The very steep gradient of polarity in the interfaces is consistent with calculated interfacial electrostatic free energy profiles of charged membranes recently reviewed by Murray et al. [26] (see their Fig. 2).

$\mathrm{X}$-Ray diffraction measurements on DOPC multilayers containing the ideally amphipathic 18-residue $\alpha$-helical peptide 18A [56] have permitted us to de-

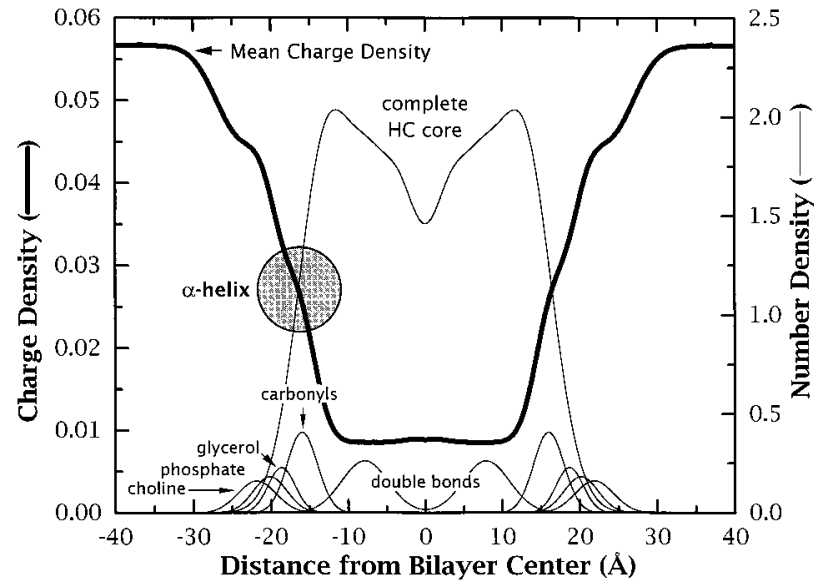

Fig. 3. Polarity profile (heavy line) and structural-group distributions from Fig. 2 (light lines). The 'polarity' of the membrane in this illustration is given as the absolute partial-charge density. Group partial-charge densities were calculated for each quasi-molecular group using the atomic partial charges of Charifson et al. [55] and the group volumes given by Wiener and White [27]. The polarity profile is obtained from the average charge density calculated by weighting the group charge densities by the number density and group volume at each position. Also shown is the schematic cross section of the amphipathic $\alpha$-helical peptide 18A (see Section 3) which is drawn to scale. Note that its location is exactly at the interface where the lipid polar groups give way to the hydrocarbon chains.

termine the precise location of the axis of the helix within the polarity profile (K. Hristova and S. White, unpublished). The axis of the 18A helix, which is parallel to the membrane surface, is located between the mean positions of the glycerol and carbonyl groups. The shaded circle in Fig. 3, representing the cross-section of $18 \mathrm{~A}$, shows that this amphipathic helix resides at approximately the mid-point of the steep decent of polarity that arises as the headgroup region gives way to the hydrocarbon region. It appears to be positioned so that its hydrophobic surface is just buried in the edge of the hydrocarbon core. (If the hydrocarbon core were a simple bulk slab of oleoyl chains, the peak of the carbonyl group would define the surface of the slab; see [27].) An interesting question that arises is how the positions of amphipathic helices will vary among the classes defined by Segrest and colleagues (see [57]). Speaking more generally, the question is how the balance of interactions in the complex interface arising from the specific amino acids in a peptide sequence determine the location and conformation of the peptide. 


\section{An experimentally determined whole-residue interfacial hydrophobicity scale}

\subsection{Experimental approach to the determination of the interfacial free energy scale}

An experimentally determined interfacial free energy scale for calculating the free energy change $\Delta G_{\text {wif }}$ associated with the transfer of an unfolded peptide chain from water to interface is an essential first step for completing the thermodynamic cycle of Fig. 1. A necessary requirement for such a scale is that it be a whole-residue scale. That is, the free energy value of a particular amino acid residue must include the peptide bond as well as the sidechain for the obvious reason that it is the entire residue that partitions into the interface. We have recently reported such a scale [34] for the 20 natural amino acids and the peptide bond derived from measurements of the partitioning small hydrophobic peptides. We have also recently reviewed the experimental methods used [35]. Because interfacial scales must describe the partitioning of unfolded peptides, the use of small peptides helped assure the absence of regular structure. We examined the partitioning of two families of peptides into large unilamellar vesicle (LUV) membranes formed from palmitoyloleoylphosphatidylcholine (POPC). One family, the 'Trppentapeptides', consisted of the complete set of hostguest peptides acetyl-WL-X-LL-OH (AcWL-X-LL) with $\mathrm{X}$ being any of the 20 natural amino acids and the other a homologous series of peptides, $\mathrm{AcWL}_{m}$ with $m=1-6$ (called the 'Leu-peptides'). The Trp-pentapeptides allowed us to determine the side-chain contributions of amino acid partitioning and the Leu-peptides the backbone $(\mathrm{CONH})$ contribution. Because the calculation of $\Delta G_{\text {wif }}$ from a hydrophobicity scale also requires additivity, the partitioning behavior of the Leu-peptides also allowed us to verify additivity, at least for peptides in the size range of our two families. In addition to partitioning between bilayers and water, we also determined the partitioning of the two families between octanol and water [58] so that a direct experimental comparison of octanol and membrane partitioning could be made. This is useful because octanol is commonly used for measurements of bulk-phase hydrophobicities [59-61].
The principal design criterion for the Trp-pentapeptides was that there be an appropriate balance between non-polar- and aqueous-phase solubility so that partition coefficients could be measured for all 20 of the natural amino acids in the $\mathrm{X}$ position. This was achieved by the relatively high non-polar composition of the peptides on the one hand and their small size and charged carboxy termini on the other. Additional design criteria, that were tested exhaustively in the course of our measurements, were that the peptides be soluble in water as monomers, lack well-defined secondary structure in aqueous and bilayer phases, and that the guest $(\mathrm{X})$ residue be in a covalent environment characteristic of unfolded proteins. The AcWL-X-LL and $\mathrm{AcWL}_{m}$ peptides satisfied all of these criteria. The $\operatorname{Trp}^{1}$ residue was in-

Table 1

Whole-residue free energies of transfer $\Delta G$ from water to POPC interface (wif) and to $n$-octanol (woct)

\begin{tabular}{|c|c|c|}
\hline Amino acid & $\Delta G_{\mathrm{wif}}(\mathrm{kcal} / \mathrm{mol})$ & $\Delta G_{\text {woct }}(\mathrm{kcal} / \mathrm{mol})$ \\
\hline Ala & $0.17 \pm 0.06$ & $0.50 \pm 0.12$ \\
\hline $\mathrm{Arg}^{+}$ & $0.81 \pm 0.11$ & $1.81 \pm 0.13$ \\
\hline Asn & $0.42 \pm 0.06$ & $0.85 \pm 0.12$ \\
\hline $\mathrm{Asp}^{-}$ & $1.23 \pm 0.07$ & $3.64 \pm 0.17$ \\
\hline $\mathrm{Asp}^{0}$ & $-0.07 \pm 0.11$ & $0.43 \pm 0.13$ \\
\hline Cys & $-0.24 \pm 0.06$ & $-0.02 \pm 0.13$ \\
\hline Gln & $0.58 \pm 0.08$ & $0.77 \pm 0.12$ \\
\hline $\mathrm{Glu}^{-}$ & $2.02 \pm 0.11$ & $3.63 \pm 0.18$ \\
\hline $\mathrm{Glu}^{0}$ & $-0.01 \pm 0.15$ & $0.11 \pm 0.12$ \\
\hline Gly & $0.01 \pm 0.05$ & $1.15 \pm 0.11$ \\
\hline $\mathrm{His}^{+}$ & $0.96 \pm 0.12$ & $2.33 \pm 0.11$ \\
\hline $\mathrm{His}^{0}$ & $0.17 \pm 0.06$ & $0.11 \pm 0.11$ \\
\hline Ile & $-0.31 \pm 0.06$ & $-1.12 \pm 0.11$ \\
\hline Leu & $-0.56 \pm 0.04$ & $-1.25 \pm 0.11$ \\
\hline Lys $^{+}$ & $0.99 \pm 0.11$ & $2.80 \pm 0.11$ \\
\hline Met & $-0.23 \pm 0.06$ & $-0.67 \pm 0.11$ \\
\hline Phe & $-1.13 \pm 0.05$ & $-1.71 \pm 0.11$ \\
\hline Pro & $0.45 \pm 0.12$ & $0.14 \pm 0.11$ \\
\hline Ser & $0.13 \pm 0.08$ & $0.46 \pm 0.11$ \\
\hline Thr & $0.14 \pm 0.06$ & $0.25 \pm 0.11$ \\
\hline Trp & $-1.85 \pm 0.06$ & $-2.09 \pm 0.11$ \\
\hline Tyr & $-0.94 \pm 0.06$ & $-0.71 \pm 0.11$ \\
\hline Val & $0.07 \pm 0.05$ & $-0.46 \pm 0.11$ \\
\hline
\end{tabular}

The values for $\Delta G_{\mathrm{wif}}$ are taken directly from [34]. The values of $\Delta G_{\text {woct }}$ are computed from data in [58] by adding the solvation energy $\Delta G_{\text {glycyl }}$ of the $-\mathrm{CH} 2-\mathrm{CONH}-$ unit $(-1.15 \pm 0.11 \mathrm{kcal} /$ $\mathrm{mol}$ [58]) to the occlusion-corrected side-chain solvation energies $\Delta \mathrm{G}_{\mathrm{X}}^{\mathrm{cor}}$ found in Table 2 of [58]. For both $\Delta G_{\text {wif }}$ and $\Delta G_{\mathrm{woct}}$, the signs have been reversed relative to those of the original publications to reflect free energies of transfer from the water phase. 
cluded because of its usefulness as a spectroscopic probe. An expected consequence of the lack of secondary structure was that the peptides would partition exclusively into the membrane interfaces. As discussed in detail elsewhere [34,35], there is little doubt that the two peptide families behave as expected.

\subsection{General features of the interface revealed by the interfacial free energy scale}

The whole-residue free energy scales determined for neutral POPC bilayers [34] and $n$-octanol [58]
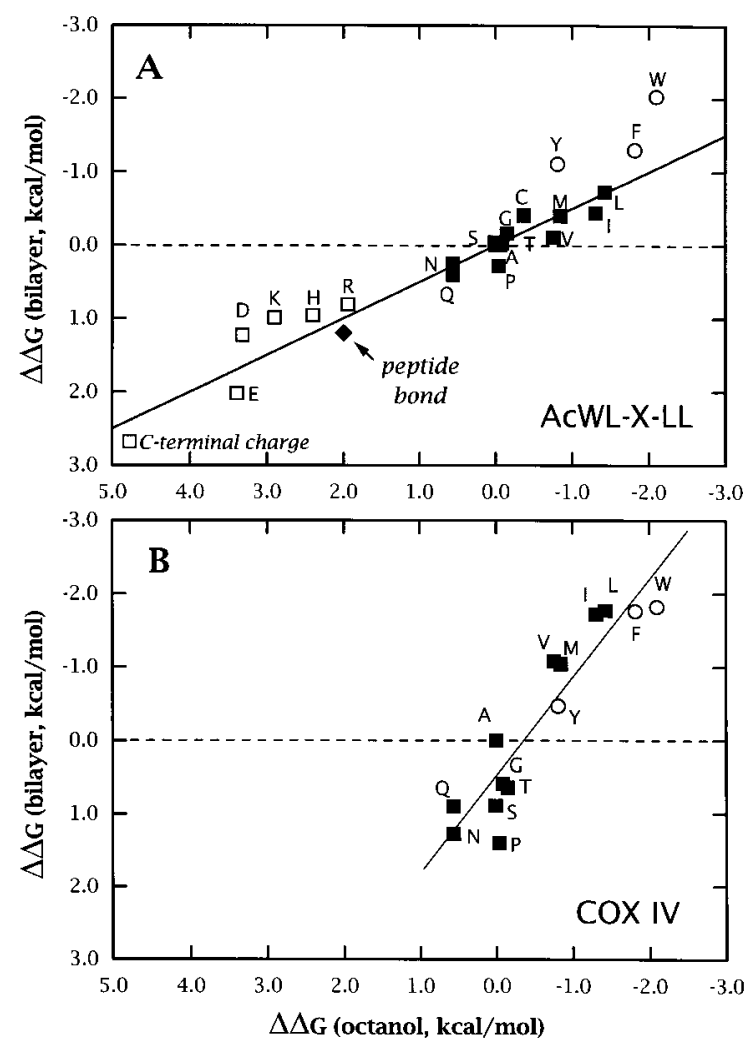

Fig. 4. Two lipid bilayer interfacial hydrophobicity scales plotted against an octanol scale determined by Wimley et al. [58]. (A) The hydrophobicities for the side-chains of the 20 natural amino acids (X) determined by Wimley and White [34] using the pentapeptides Ac-WL-X-LL. This plot is reproduced from Fig. $3 b$ of Wimley and White with permission. The straight line has a slope of 0.5 , indicating that the interfacial hydrophobicity is equivalent to about one-half that of octanol (see Section 4). (B) The hydrophobicities for the side-chains of 14 natural amino acids in the $\cdot \mathrm{R}_{5}-\mathrm{Q}-\mathrm{X}-\mathrm{I}-\mathrm{R}_{9} \cdot$ region of the 25 -residue presequence of subunit IV of cytochrome $c$ oxidase (COX IV) determined by Thorgeirsson et al. [75]. The slope of the straight line is 1.3 , suggesting that the guest residues of the COX IV peptide sense a more non-polar environment (see Section 5). are summarized in Table 1. The bilayer-interface and octanol hydrophobicities are strongly correlated, but the bilayer values are generally about one-half those observed for octanol. This is shown graphically in Fig. 4A where we have plotted the bilayer values relative to Ala against the equivalent octanol values. Also included are the values for the peptide bond $(\mathrm{CONH})$ and the charged carboxyl terminus. A similar plot of the whole-peptide free energies of transfer for the Leu-peptides gave straight line with a slope of 0.49 (see [34], Fig. 3A). The straight line with this slope added to the data of Fig. 4A shows that it roughly describes the relative contributions of the side-chain, carboxyl terminus, and peptide bond groups. The deviations from the line reveal interesting differences between bilayer and octanol partitioning. For example, the $\beta$-branched hydrophobes Val and Ile are much less hydrophobic in the bilayer than expected, whereas the aromatics are more hydrophobic. The observation that interfacial hydrophobicity is proportional to octanol hydrophobicity supports the earlier conclusion that membrane partitioning is driven mainly by the hydrophobic effect [25]. However, the slope of 0.5 of the solid line and the fluctuations of the points around it reveal that the details of the interactions with the membrane-water interface contribute important effects.

Jacobs and White [25] found that the non-polar solvation parameter of their five tripeptides, $12 \mathrm{cal} /$ $\mathrm{mol} / \AA^{2}$, was about one-half the value observed for partitioning into bulk organic phases $(\sim 22 \mathrm{cal} / \mathrm{mol} /$ $\AA^{2}$ ), consistent with the slope of the line in Fig. 4A. They suggested that the smaller solvation parameter might mean that either it is a general property of the complex interfacial region or the non-polar surface is about $50 \%$ buried in the outer edge of the hydrocarbon core. Both effects may be operating, but the data suggest that the first explanation may be more likely because the solvation parameters of charged, polar, and non-polar moieties alike are about onehalf the values observed for octanol partitioning. This view is certainly consistent with the structural image and polarity profile of Figs. 2 and 3. A simple, but probably incomplete, explanation of the $50 \%$ effect is that the average dielectric coefficient $(\varepsilon)$ of the interface is somewhat higher than that of octanol. The calculations of Flewelling and Hubbell [62] indicate that an increase of $\varepsilon$ from about 12 in hy- 
drated octanol to 18 in the bilayer interface would explain the reduction of about one-half in the interfacial transfer free energy of the carboxyl terminus (2.68 kcal $/ \mathrm{mol},[34])$ compared to the octanol value (4.78 kcal/mol, [58]). Such simple explanations, based solely on bulk-phase properties, are probably not warranted, however, because structural studies [63] of octanol indicate that it too is a complex interfacial phase. It would otherwise be difficult to explain why the non-polar solvation parameters for alkanes $(\varepsilon \sim 2)$ and octanol $(\varepsilon \sim 12)$ are the same [58]. Whatever the explanation may be for the $50 \%$ effect, it is clear that the phosphatidylcholine interface has significant non-polar character, consistent with the image of Fig. 2.

\subsection{Specific features of the interfacial free energies}

The relative values of the free energies of the 20 amino acids do not form a simple continuum. Although the charged residues are at one end of the scale and the hydrophobes at the other, indicating that the hydrophobic effect plays a central role in partitioning, a closer examination of Table 1 and Fig. 4A reveals that the 20 amino acids fall roughly into three ranks of importance. The dominating first rank is comprised of aromatic residues that have very favorable free energies and the charged residues which have large unfavorable free energies. The second-rank contributors to partitioning are the carboxyamide side-chains Gln and Asn which are unfavorable by $\sim 0.5 \mathrm{kcal} / \mathrm{mol}$ and Leu which is favorable by $\sim 0.5 \mathrm{kcal} / \mathrm{mol}$. The bottom third-rank residues make relatively small net contributions $(\sim 0.25 \mathrm{kcal} / \mathrm{mol})$. Surprisingly, Ala, Val, Ile, and Met fall into this rank, indicating the lack of a simple linear relationship between octanol and interface partitioning.

The interfacial hydrophobicities of all of the charged side-chains are approximately equal, contrary to some expectations. Interfacial partitioning of Lys and Arg have been assumed to be relatively favorable because of the possibility of their methylenes interacting hydrophobically with the membrane interface while their charged moieties interact favorably with the aqueous environment $[25,64,65]$. The data of Fig. 4A indicate clearly that this so called 'snorkel effect' does not occur for the pentapeptides.
This is consistent with the fact that the membrane interface occupies a thickness of at least $15 \AA$ (Fig. 2), compared to the $6 \AA$ length of an Arg and Lys side-chains.

The high interfacial hydrophobicity of the aromatics is disproportionally large relative to their hydrophobicity in octanol, in agreement with studies of the exceptionally strong partitioning of indole and indole analogs into the membrane interface [18]. This strengthens the idea that there are special interactions there which are important in the structure and function of channel-forming peptides [66-68] and membrane proteins $[18,69,70]$ since Trp and Tyr are found with a high frequency near the ends of transmembrane domains that are expected to be in the membrane interface. The suggestion has been made [70] that Trp and Tyr prefer the interface because of their amphipathic nature, the idea being that the aromatic rings reside in the hydrocarbon core with their polar $-\mathrm{NH}$ or $-\mathrm{OH}$ groups forming hydrogen bonds with lipid carbonyls. However, bilayer partitioning data for several indole compounds [18], the transbilayer distribution of the Trp of A-WA-O- $t$-bu determined by neutron diffraction (Fig. 2), and preliminary NMR measurements [71] suggest that the interaction is probably not that simple and is likely to be novel. Close examination of the Trp distribution in Fig. 2 indicates that Trp is not constrained mostly to the hydrocarbon core. Rather, it is much more closely associated with the glycerophosphocholine group. The bilayer partitioning data for indole compounds [18] reveal small, but important, differences in the values of $\Delta G_{\text {wif }}$ for $N$-methylindole $(-8.1 \mathrm{kcal} / \mathrm{mol})$ and $3-\mathrm{methylindole}(-8.6 \mathrm{kcal} / \mathrm{mol})$. Given the observation $[23,24]$ that the partitioning of $N$-methylindole into cyclohexane is more favorable by $1.2 \mathrm{kcal} / \mathrm{mol}$ than the partitioning of 3-methylindole because of the loss of the hydrogen bonding potential of the imide nitrogen, one might expect higher partitioning of the $N$-methyl compound. Furthermore, if the indole were being 'held' at the interface by a COO $\cdots \cdot \cdot \mathrm{HN} \mathrm{H}$-bond, then one would expect the $N$-methyl compound to have a much more favorable interaction with the hydrocarbon core and move deeper into it. Heat capacity measurements [18] suggest that the opposite is true and NMR measurements show only minor differences in the dispositions of the two compounds in the interface [71]. 


\subsection{Partitioning-folding coupling}

Broadly speaking, as discussed earlier, the interfacial free energy values for the 20 natural amino acids fall into three distinct classes: all of the charged residues, the carboxyl terminus, and the peptide bond are highly unfavorable, the aromatics are highly favorable, and the remaining residues make smaller contributions. Very important is the fact that the cost of transferring a peptide bond into the membrane interface, $+1.2 \mathrm{kcal} / \mathrm{mol}$, is as costly as transferring a charged side-chain. Because its magnitude is about the same size as the magnitudes of the more hydrophobic side-chains, it essentially dominates the whole-residue values. This high cost must figure prominently in the formation of secondary structure in membrane interfaces. As noted earlier (Section 1), the participation of the peptide bond in H-bonds greatly reduces its cost of partitioning into non-polar phases. We have suggested that this effect operates in the membrane interface and is important for the promotion of secondary structure observed when small toxins [72], antimicrobial peptides [73], and signal sequences [74] partition into membrane interfaces.
We refer to this process as partitioning-folding coupling [34].

A classic example of partitioning-folding coupling is seen in the partitioning of melittin (26 residues) into phosphatidylcholine bilayers [35,36]. At low concentrations in aqueous phases of low ionic strength, melittin is monomeric and is largely random coil with low residual helicity $(\sim 10 \%)$ as judged by circular dichroism spectroscopy. Upon partitioning into the bilayer interface, however, melittin adopts a highly helical conformation $(\sim 85 \%$, corresponding to about 20 residues). Titration of melittin solutions with phosphatidylcholine vesicles produces a set of spectra whose ellipticity at $222 \mathrm{~nm}$ decreases in proportion to the amount of melittin bound. Importantly, the collection of spectra reveal a very distinct isodichroic point at about $200 \mathrm{~nm}$, indicative of a two-state transition in which there are only two significantly populated states: monomeric melittin in the aqueous phase with low helicity and membrane-bound melittin with high helicity (see Fig. 1a of [36] and Fig. 1 of [35]). Plots of ellipticity at $222 \mathrm{~nm}$ against lipid concentration permit the partition coefficient from which a free energy of

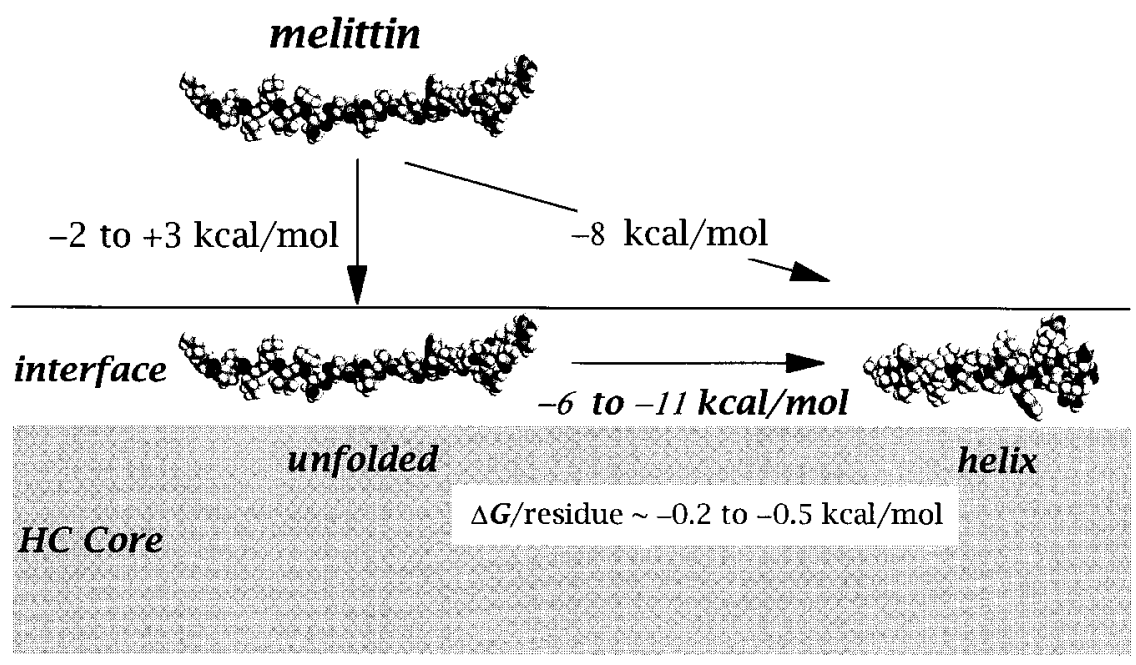

interface

Fig. 5. A thermodynamic cycle for the partitioning and folding of melittin into the bilayer interface based on work published by White et al. [35] that describes partitioning-folding coupling. Circular dichroism spectroscopy indicates that only the folded membrane-bound states and the unfolded aqueous states are occupied to a significant extent [35,36]. The whole-residue interfacial hydrophobicity scale of Wimley and White [34] permits the partitioning free energy of the unfolded form to be estimated. The free energy reduction of $0.2-0.5 \mathrm{kcal} / \mathrm{mol}$ arises to a major extent from the reduction in the cost of partitioning the peptide bond caused by hydrogen bonding (see Section 4.4). 
transfer $\Delta G_{\text {wif }}+\Delta G_{\text {fif }} \approx 8 \mathrm{kcal} / \mathrm{mol}$ can be established [35].

The significance of this coupling of melittin folding to partitioning is that two equilibria contribute simultaneously to the observable process: the monomer partitioning equilibrium and the equilibrium between folded and unfolded melittin bound to the interface. This is shown schematically in Fig. 5 as a thermodynamic cycle. The populations of each state must be independently determined in order to understand the thermodynamics of the coupling of folding to partitioning. However, the bound random-coil state of melittin cannot be seen by CD spectroscopy and therefore the membrane concentration of the unfolded form cannot be determined. The usefulness of the interfacial hydrophobicity scale now becomes apparent because it can be used in Fig. 5 to estimate the free energy of the partitioning of unfolded melittin. Depending upon assumptions about the disposition of the six highly polar residues at the C-terminus, $\Delta G_{\text {wif }}^{\mathrm{v}}$ is estimated to be between -2 and +3 $\mathrm{kcal} / \mathrm{mol}$, meaning that $\Delta G_{\text {fif }}$ is between -6 to -11 $\mathrm{kcal} / \mathrm{mol}$. (As a reference value, it useful to note that a $\Delta G$ of $-6 \mathrm{kcal} / \mathrm{mol}$ corresponds to the folded state being about 25000 times more populated than the unfolded state.) In the absence of any other effects, this folding can be explained by a modest reduction, $0.2-0.5 \mathrm{kcal} / \mathrm{mol}$, in the cost of partitioning each peptide bond. But, other processes are probably also important as indicated by the partial burying of the 18A amphipathic peptide in the hydrocarbon interface (Fig. 3).

\section{Complexities of interfacial partitioning}

The chemically heterogeneous and highly anisotropic interfacial layers that separate the bulk water phase from the hydrocarbon core (Fig. 2) defy any attempt to describe them with a single set of parameters, or with a unique hydrophobicity scale, because their physicochemical properties vary so dramatically with depth (Fig. 3). Instead, there is probably a continuum of 'hydrophobicities' that bridge the gap between water and the hydrocarbon core. Peptides partitioning into this variable interface, of course, will follow this physicochemical gradient into the bilayer to an equilibrium depth and disposition that yields the largest reduction in free energy. That is, the final location in the membrane will depend on the balance between hydrophobic, electrostatic, bilayer effects, and other interactions and on the conformational states available to the peptide. Weakly binding peptides without secondary structure, such as the small peptides that form the basis for our whole-residue interfacial hydrophobicity scale, may be nearer to the aqueous edge of the interface, as shown by the neutron diffraction study of A-W-A-O-t-bu (Fig. 2), because of the high cost of partitioning the peptide bond. In this light, our interfacial hydrophobicity scale may represent a region of the bilayer interface with lower methylene content.

What is the nature of this interfacial environment? As we discussed earlier, there is a remarkable correlation between the experimentally determined interface and octanol hydrophobicities of non-polar, polar, and charged groups because, overall, the data are described well by a single line with a slope of 0.5 . This suggests that for our small peptides, all of the favorable and unfavorable free energy contributions in the bilayer are approximately one half of the respective value in bulk octanol. Although we do not understand this result on a molecular or physicochemical level, it is nonetheless an encouraging result in the sense that it suggests that it may be empirically useful to describe the membrane interface in broad, relatively simple phenomenological terms related to bulk solvent properties.

Support for this idea is provided in Fig. 4B where the results of the bilayer partitioning studies of Thorgeirsson et al. [75] are shown plotted against the pentapeptide octanol results. These partitioning data come from a 25 -residue host-guest peptide derived from the leader sequence of subunit IV of cytochrome $c$ oxidase (COX IV). As for our pentapeptides, COX IV was shown to lack a significant amount of well-defined secondary structure when bound to membranes composed of mixtures of POPC and POPG. We chose in plotting the data of Thorgeirsson et al. to use our pentapeptide octanolpartitioning values as the reference scale rather than the commonly used $\mathrm{N}$-acetyl-amino-acid amide values [59] because the occlusion [58] of the guest residue ( $\mathrm{X}$ in $\cdot \mathrm{R}_{5}$-Q-X-I- $\left.\mathrm{R}_{9} \cdot\right)$ and its neighbors is likely to be similar to that of the guest residue in AcWL-XLL. Fig. 4B shows that the COX IV hydrophobicity 
scale is also strongly correlated with octanol hydrophobicity. Interestingly, however, the slope is much larger, $\sim 1.3$ (Thorgeirsson et al. obtained $\sim 1$ using the Ac-amino-acid amide data), suggesting a considerably more hydrophobic interfacial environment for the guest site in the COX IV peptides compared to the pentapeptides.

The difference between the pentapeptide and COX IV results indicates how little we know at this time about the details of the interfacial interactions and raises fundamental questions. The simple-minded explanation for the difference is that the equilibrium positions for the two classes of peptides in the interface are different, causing a difference in the amount of hydrocarbon core visited. Although the 'polarity' gradient shown in Fig. 3 permits this possibility, the existence of 'open' peptide bonds in unfolded peptides should prohibit deep penetration, regardless of the size of the peptide. Are there differences in secondary structure that cannot be detected? What is the effect of lipid composition? How are the lipids rearranged in the vicinity of the peptides? Are the guest residues really sampling a largely lipid environment or are they sampling a mixed lipid-peptide environment created by lipid rearrangements? The answers to these and many other questions must await new experimental insights. For present, the questions serve to remind us of the complex nature of peptide interactions at bilayer interfaces.

\section{Acknowledgements}

This work was supported in part by grant GM46823 from the National Institute of General Medical Sciences. We thank Drs. Stuart McLaughlin and David Cafiso for useful discussions.

\section{References}

[1] K.A. Dill, Biochemistry 29 (1990) 7133-7155.

[2] T.E. Creighton, Protein Folding, W.H. Freeman, New York, 1992, pp. 1-547.

[3] A. Chakrabartty, R.L. Baldwin, Adv. Protein Chem. 46 (1995) 141-176.

[4] E. Freire, Annu. Rev. Biophys. Biomol. Struct. 24 (1995) $141-165$.
[5] G.I. Makhatadze, P.L. Privalov, Adv. Protein Chem. 47 (1995) 307-425.

[6] J.-L. Popot, Curr. Opin. Struct. Biol. 3 (1993) 532-540.

[7] S.H. White, W.C. Wimley, Curr. Opin. Struct. Biol. 4 (1994) 79-86.

[8] S.H. White, Membrane Protein Structure: Experimental Approaches, Oxford University Press, New York, 1994, pp. 1395.

[9] D.C. Rees, A.J. Chirino, K.-H. Kim, H. Komiya, in: S.H. White, (Ed.), Membrane Protein Structure: Experimental Approaches, Oxford University Press, New York, 1994, pp. 3-26.

[10] F.M. Richards, in: T.E. Creighton (Ed.), Protein Folding W.H. Freeman, New York, 1992, pp. 1-58.

[11] P.L. Privalov, in: T.E. Creighton (Ed.), Protein Folding, W.H. Freeman, New York, 1992, pp. 83-126.

[12] C.N. Pace, Methods Enzymol. 131 (1986) 266-280.

[13] T. Haltia, E. Freire, Biochim. Biophys. Acta 1241 (1995) 295-322.

[14] D.M. Engelman, T.A. Steitz, A. Goldman, Annu. Rev. Biophys. Biophys. Chem. 15 (1986) 321-353.

[15] M.A. Roseman, J. Mol. Biol. 201 (1988) 621-625.

[16] N. Ben-Tal, A. Ben-Shaul, A. Nicholls, B. Honig, Biophys. J. 70 (1996) 1803-1812.

[17] S.H. White, in: S.H. White (Ed.), Membrane Protein Structure: Experimental Approaches, Oxford University Press, New York, 1994, pp. 97-124.

[18] W.C. Wimley, S.H. White, Biochemistry 32 (1993) 63076312.

[19] C.-H. Huang, J.P. Charlton, Biochemistry 11 (1972) 735740.

[20] Y.K. Levine, M.H.F. Wilkins, Nat. New Biol. 230 (1971) 69-72.

[21] J. Seelig, P. Ganz, Biochemistry 30 (1991) 9354-9359.

[22] G. Beschiaschvili, J. Seelig, Biochemistry 31 (1992) 10044 10053.

[23] W.C. Wimley, S.H. White, Biochemistry 31 (1992) 1281312818.

[24] W.C. Wimley, S.H. White, Biochemistry 32 (1993) 9262.

[25] R.E. Jacobs, S.H. White, Biochemistry 28 (1989) 34213437.

[26] D. Murray, N. Ben-Tal, B. Honig, S. McLaughlin, Structure 5 (1997) 985-989.

[27] M.C. Wiener, S.H. White, Biophys. J. 61 (1992) 434 447.

[28] T.S. Moll, T.E. Thompson, Biochemistry 33 (1994) 1546915482.

[29] M. Soekarjo, M. Eisenhawer, A. Kuhn, H. Vogel, Biochemistry 35 (1996) 1232-1241.

[30] J.F. Hunt, P. Rath, K.J. Rothschild, D.M. Engelman, Biochemistry 36 (1997) 15177-15192.

[31] J.F. Hunt, T.N. Earnest, O. Bousché, K. Kalghatgi, K. Reilly, C. Horváth, K.J. Rothschild, D.M. Engelman, Biochemistry 36 (1997) 15156-15176.

[32] N. Grigorieff, T.A. Ceska, K.H. Downing, J.M. Baldwin, R. Henderson, J. Mol. Biol. 259 (1996) 393-421. 
[33] E. Pebay-Peyroula, G. Rummel, J.P. Rosenbusch, E.M. Landau, Science 277 (1997) 1676-1681.

[34] W.C. Wimley, S.H. White, Nat. Struct. Biol. 3 (1996) 842 848.

[35] S.H. White, W.C. Wimley, A.S. Ladokhin, K. Hristova, Methods Enzymol. 295 (1998) 62-87.

[36] H. Vogel, FEBS Lett. 134 (1981) 37-42.

[37] L.A. Chung, T.E. Thompson, Biochemistry 35 (1996) 11343 11354.

[38] S.-C. Li, C.M. Deber, Nat. Struct. Biol. 1 (1994) 368-373.

[39] C.M. Deber, S.-C. Li, Biopolymers 37 (1995) 295-318.

[40] S.E. Blondelle, B. Forood, R.A. Houghten, E. Pérez-Payá, Biopolymers 42 (1997) 489-498.

[41] M.C. Wiener, S.H. White, Biophys. J. 59 (1991) 162-173.

[42] M.C. Wiener, S.H. White, Biophys. J. 59 (1991) 174-185.

[43] M.C. Wiener, S.H. White, Biochemistry 30 (1991) $6997-$ 7008.

[44] M.C. Wiener, G.I. King, S.H. White, Biophys. J. 60 (1991) 568-576.

[45] M.C. Wiener, S.H. White, Biophys. J. 61 (1992) 428-433.

[46] S.H. White, M.C. Wiener, in: E.A. Disalvo, S.A. Simon (Eds.), Permeability and Stability of Lipid Bilayers, CRC Press, Boca Raton, 1995, pp. 1-19.

[47] S.H. White, M.C. Wiener, in: K.M. Merz, B. Roux (Eds.), Membrane Structure and Dynamics, 1996, Birkhäuser, Boston, pp. 127-144.

[48] P.B. Hitchcock, R. Mason, K.M. Thomas, G.G. Shipley, Proc. Natl. Acad. Sci. USA 71 (1974) 3036-3040.

[49] I. Pascher, M. Lundmark, P.-G. Nyholm, S. Sundell, Biochim. Biophys. Acta 1113 (1992) 339-373.

[50] T.J. McIntosh, P.W. Holloway, Biochemistry 26 (1987) 1783-1788.

[51] N.P. Franks, T. Arunachalam, E. Caspi, Nature 276 (1978) 530-532.

[52] D.L. Worcester, N.P. Franks, J. Mol. Biol. 100 (1976) 359378.

[53] G. Büldt, H.U. Gally, A. Seelig, J. Seelig, G. Zaccai, Nature 271 (1978) 182-184.

[54] K. Hristova, S.H. White, Biophys. J., in press.
[55] P.S. Charifson, R.G. Hiskey, L.G. Pedersen, J. Comput. Chem. 11 (1997) 1181-1186.

[56] V.K. Mishra, M.N. Palgunachari, J.P. Segrest, G.M. Anantharamaiah, J. Biol. Chem. 269 (1994) 7185-7191.

[57] J.P. Segrest, H. Deloof, J.G. Dohlman, C.G. Brouillette, G.M. Anantharamaiah, Proteins 8 (1990) 103-117.

[58] W.C. Wimley, T.P. Creamer, S.H. White, Biochemistry 35 (1996) 5109-5124.

[59] J.-L. Fauchère, V. Pliska, Eur. J. Med. Chem.-Chim. Ther. 18 (1983) 369-375.

[60] A. Radzicka, R. Wolfenden, Biochemistry 27 (1988) 1664 1670.

[61] A. Kim, F.C. Szoka Jr., Pharmaceut. Res. 9 (1992) 504-514.

[62] R.F. Flewelling, W.L. Hubbell, Biophys. J. 49 (1986) 531540.

[63] N.P. Franks, M.H. Abraham, W.R. Lieb, J. Pharm. Sci. 82 (1993) 466-470.

[64] J.P. Segrest, M.K. Jones, H. Deloof, C.G. Brouillette, Y.V. Venkatachalapathi, G.M. Anantharamaiah, J. Lipid Res. 33 (1992) 141-166.

[65] R. Schwyzer, Chemtracts-Biochem. Mol. Biol. 3 (1992) 347379.

[66] J.A. Killian, J.W. Timmermans, S. Keur, B. de Kruijff, Biochim. Biophys. Acta 820 (1985) 154.

[67] W. Hu, K.C. Lee, T.A. Cross, Biochemistry 32 (1993) 70357047.

[68] R.E. Koeppe, J.A. Killian, D.V. Greathouse, Biophys. J. 66 (1994) 14-24.

[69] C. Landolt-Marticorena, K.A. Williams, C.M. Deber, R.A.F. Reithmeier, J. Mol. Biol. 229 (1993) 602-608.

[70] M. Schiffer, C.H. Chang, F.J. Stevens, Protein Eng. 5 (1992) 213-214.

[71] W.-M. Yau, W.C. Wimley, K. Gawrisch, S.H. White, Biochemistry (1998), in press.

[72] Y. Shai, Toxicology 87 (1994) 109-129.

[73] W.L. Maloy, U.P. Kari, Biopolymers 37 (1995) 105-122.

[74] L.M. Gierasch, Biochemistry 28 (1989) 923-929.

[75] T.E. Thorgeirsson, C.J. Russell, D.S. King, Y.-K. Shin, Biochemistry 35 (1996) 1803-1809. 